\title{
Healing period after open high tibial osteotomy and related factors: Can we really say that it is long?
}

\author{
Masamichi Yokoyama*, Yasuhiro Nakamura, Toru Onishi, Koji Hirano and Motoyuki Doi
}

\begin{abstract}
High tibial osteotomy (HTO) is a general procedure for the treatment of degenerative gonarthrosis. In recent years, it has been reported that opening wedge high tibial osteotomy (OWHTO) has become widespread with good results. Despite these facts, HTOs tend to be avoided due to the need for long-term postoperative treatment. To investigate the treatment period for total recovery (healing period) after OWHTO and the factors affecting it. There were 47 cases of medial type degenerative gonarthrosis who underwent OWHTO from 2008 through 2011. The definition of the healing period was based on the time-dependent changes of the Japanese Orthopaedic Association score, focusing especially on pain on walking and pain on ascending/descending stairs. This score was defined as the Ability score. In this study, the healing period ended when the Ability score reached its maximum or when it showed a perfect score. Patients' characteristics were examined to determine their effect on the healing period. The Ability score was $36.7 \pm 10.1$ (mean \pm SD) before surgery and $51.6 \pm 5.412$ months after OWHTO. The healing period was $6.3 \pm 3.9$ months. Factors correlated with a longer healing period included female sex (correlation coefficient -0.35 ) and high BMI (correlation coefficient 0.33). Our study suggested that the healing period after OWHTO is approximately 6 months, and patients' BMI and sex appear to be related to this period. This information is expected to be helpful for counseling patients undergoing treatment for gonarthrosis.

Level of evidence Therapeutic study, Level IV.
\end{abstract}

Keywords: Degenerative gonarthrosis, Surgical management, High tibial osteotomy, Treatment period

\section{Background}

High tibial osteotomy (HTO) is a major surgical procedure that improves the condition of the knee joint. In recent years, opening wedge high tibial osteotomy (OWHTO) has also been increasingly used. However, the number of HTOs performed has not increased much, even though there are increasing numbers of patients with degenerative gonarthrosis. The HTO procedure is technically difficult to perform, therefore, highly experienced surgeons are needed for successful outcomes. Another reason is the long treatment period for total recovery after HTO. The duration of the treatment period has become an important issue, and this time period has

*Correspondence: yoko2974@me.com

Department Orthopedics, Okayama Kyokuto Hospital, Kurata 567-1,

Naka-ku, Okayama City, Okayama 703-8265, Japan an effect even on the choice of surgical method. However, the details of the process for obtaining good results in the short-term after HTO have not been well reported. Therefore, one must question whether there is any definitive evidence to suggest that healing takes a long time after HTO.

Thus, this study aimed to determine the actual period for total recovery (healing period) and to identify factors affecting the length of the healing period after the procedure.

\section{Patients and methods}

A total of 47 cases (13 males, 34 females; mean age 59.8 years) who underwent OWHTO for medial type degenerative gonarthrosis that were unresponsive to conservative treatment between 2008 and 2011 were evaluated. In fact, OWHTO was carried out for 62 cases

\section{贷 Springer}

(c) 2016 Yokoyama et al. This article is distributed under the terms of the Creative Commons Attribution 4.0 International License (http://creativecommons.org/licenses/by/4.0/), which permits unrestricted use, distribution, and reproduction in any medium, provided you give appropriate credit to the original author(s) and the source, provide a link to the Creative Commons license, and indicate if changes were made. 
in total, but cases of simultaneous bilateral knee joint surgery and cases in which each patient's femoro-tibial angle (FTA) was not corrected to within the general target angle of $167^{\circ}-172^{\circ}$ after the operation were excluded. FTA should be around $170^{\circ}$ after OWHTO for better outcomes, and the surgery is planned to correct FTA to that degree. Therefore, cases with FTA outside of this range were excluded.

All patients had medial type degenerative gonarthrosis with grade 2, 3, or 4 of the Kellgren-Lawrence (K-L) classification (Table 1).

The following preoperative characteristics of the patients were evaluated: age; sex; K-L classification; body mass index (BMI); FTA measured on whole standing lower limb X-rays; mechanical axis percentage (\%MA) where the Mikulicz line goes through the tibial plateau in the range of 0 in the internal edge to 100 in the external edge on the X-ray; posterior tibial slope (PTS); and JOA score. The FTA, \%MA, and PTS were also evaluated postoperatively. The tibia was opened to the angle that was the same as the wedge-shaped spacer used in the operation. This was evaluated as the opening angle. Furthermore, for detailed evaluation, the JOA score was evaluated by physiotherapists 1.5 months after OWHTO and then every 3 months for 1 year.

The surgery was planned to correct the weight-bearing line of the lower limb to be about $63 \%$, the proper angle for the mechanical axis, called Fujisawa's point. The tibia was opened with a wedged spacer set at this angle, and a United States Food and Drug Administration (FDA)approved OSferion60 ( $\beta$ tricalcium phosphate, Olympus Terumo Biomaterial Corporation, Tokyo, Japan) of the same shape was placed inside. Then, internal fixation was completed with an FDA-approved Plating system (TOMOFIX system, DePuy Synthes Companies, Zuchwil, Switzerland) (Fig. 1). All cases were treated with this surgical technique by one surgeon.

Rehabilitation started on the day after OWHTO with range of motion (ROM) training. In addition, from the 2nd day after the surgery, patients started walking

Table 1 Data of examined patients

\begin{tabular}{ll}
\hline Cases & 47 \\
Age $\left(\right.$ mean $\pm \mathrm{SD}^{\circ}($ range $\left.)\right)$ & $59.8 \pm 9.5(35-73)$ \\
Sex & 13 Males \\
& 34 Females \\
K-L classification & 18 Grade II \\
& 28 Grade III \\
& 1 Grade IV \\
BMI $\left(\mathrm{kg} / \mathrm{m}^{2}\right)\left(\right.$ mean $\pm \mathrm{SD}^{\circ}($ range $\left.)\right)$ & $26.2 \pm 3.4(20.6-37.0)$ \\
\hline
\end{tabular}

$S D$ standard deviation, $K-L$ Kellgren Lawrence, $B M I$ body mass index training with a load. This load was not restricted, but was chosen according to the degree of each patient's pain. The degree of pain was observed by the change of the JOA score with time. It was mainly evaluated on the basis of two JOA score domains, pain on walking and pain on ascending/descending stairs. This was defined as the Ability score. Two types of end points were then set in order to determine the healing period: either the score reached the perfect score, or it showed no further improvement (Fig. 2). According to this definition, all cases were divided into two groups for comparison, the Early Cured group (fully improved within 9 months) and the Late Cured group (required 12 months for healing).

This study was carried out according to the ethical requirements of our hospital.

\section{Results}

\section{Improvement and healing period}

The average data (mean \pm SD) of the 47 cases are reported. Comparing preoperative and 1-year postoperative values, FTA was corrected from $178 \pm 2.4^{\circ}$ to $168.9 \pm 2.2^{\circ}$. The values of \%MA and PTS also showed improvement, from $24.9 \% \pm 11.6 \%$ to $66.7 \% \pm 9.7 \%$ and from $10.3 \pm 3.0^{\circ}$ to $13.8 \pm 3.3^{\circ}$, respectively. The total JOA score increased from $71.7 \pm 12.2$ to $93.1 \pm 7.4$, and the Ability score improved from $36.7 \pm 10.1$ to $51.6 \pm 5.4$ (Table 2). The average healing period was 6.3 months; 38 cases were in the Early Cured group, while 9 cases were in the Late Cured group (Table 3).

\section{Statistical analysis of the Ability score}

Considering the changes of the Ability score over time, there was a trend to improvement at the 1.5-month evaluation (paired $t$ test, $\mathrm{P}<0.001$ ), with a significant improvement at 3 months after surgery (paired $t$ test, $\mathrm{P}<0.001$ ). There continued to be moderate but meaningful improvements at the 6- and 9-month evaluations (paired $t$ test, $\mathrm{P}=0.044$ at the 6th month, $\mathrm{P}=0.014$ at the 9th month, Fig. 3).

\section{Comparing data of the two groups (Table 3)}

Comparing the preoperative characteristics between the Early Cured group and the Late Cured group, the average BMI was significantly higher in the Late Cured group $(28.3 \pm 0.4$ SE vs. $25.7 \pm 1.6 \mathrm{SE}$; Student's $t$ test, $\mathrm{P}=0.031$, Fig. 4). The Ability score was also higher in the Early Cured group, but the difference was not significant. Other preoperative characteristics, including age, $\mathrm{K}-\mathrm{L}$ classification, FTA, \%MA, and PTS, showed no significant differences (Fig. 4). As for the postoperative examination, no differences were seen in the actual opening angle, FTA, \%MA, and PTS (Fig. 5). 

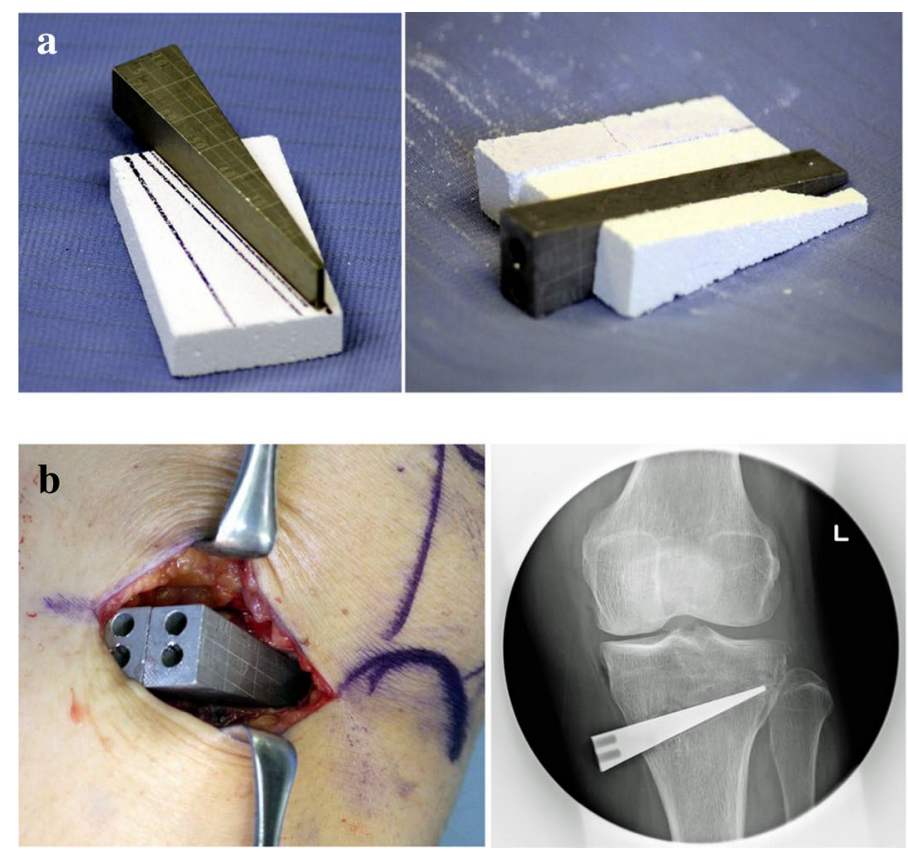

Fig. 1 a A spacer is prepared to achieve the correction angle, and an OSferion is formed in the same shape as the spacer. $\mathbf{b}$ The cut tibia is opened with the spacer, and its condition is checked using fluoroscopy. c The spacer in the opening space is substituted by the same-shaped OSferion and is fixed with a TomoFix plate

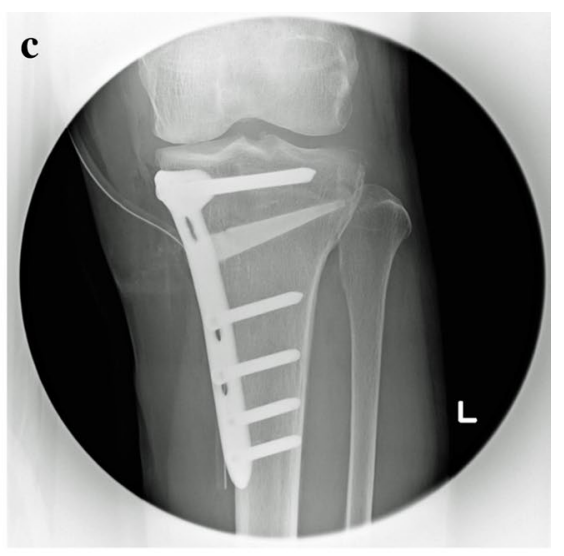

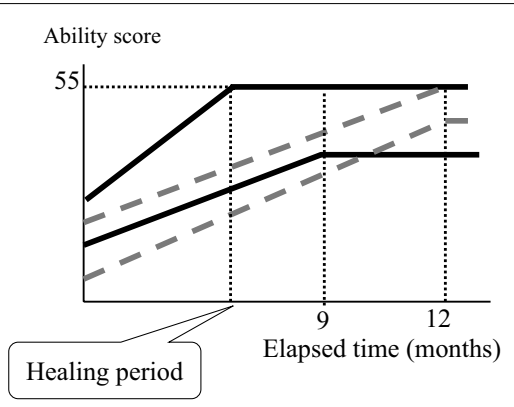

Fig. 2 Patients in the Early Cured group heal within 9 months (shown as the solid line) with the defined end point, which shows a full score (55 points) or no further increase in the Ability score. The Late Cured group patients require 12 months to heal (shown as a dotted line)

Table 2 Changes before and after OWHTO in lower limb alignment and clinical condition

\begin{tabular}{lcc}
\hline & $\begin{array}{l}\text { Before HTO } \\
\text { (mean } \pm \text { SD }\end{array}$ & $\begin{array}{l}\text { 1 range) } \\
\text { (mean } \pm \text { SD }^{\circ} \text { (range) }\end{array}$ \\
\hline FTA & $178.0 \pm 2.4(172-185)$ & $168.9 \pm 2.2(165-174)$ \\
$\% M A$ & $24.9 \pm 11.6(0-50)$ & $66.7 \pm 9.7(47-88)$ \\
PTS & $10.3 \pm 3.0(4-16)$ & $13.8 \pm 3.3(8-21)$ \\
JOA score & $71.7 \pm 12.2(45-95)$ & $93.1 \pm 7.4(70-100)$ \\
Ability score & $36.7 \pm 10.1(10-55)$ & $51.6 \pm 5.4(35-55)$ \\
\hline
\end{tabular}

HTO high tibial osteotomy, OWHTO opening wedge HTO, SD standard deviation, FTA femorotibial angle, MA mechanical axis, PTS posterior tibial slope, JOA Japanese Orthopaedic Association

\section{Correlations}

With respect to the correlation between the healing period and each factor examined, the healing period had a positive correlation with BMI (Pearson's correlation coefficient: 0.32 ). In other words, a higher BMI value was associated with longer healing time (Fig. 6a). The healing period and patients' sex were also correlated (Pearson's correlation coefficient: 0.35 ). The healing period was significantly shorter in men $(4.1 \pm 0.9 \mathrm{SE}$ months) than in women $(7.1 \pm 0.7$ (SE) months; Student's $t$ test, $\mathrm{P}=0.018$ ) (Fig. 6b).

\section{Discussion}

Surgeons seem reluctant to use HTO as a procedure due to the long healing period, although they make this decision primarily based on their own experience. In the present study, the healing period and the process involved after OWHTO were examined. Understanding these could be a strong motivation to recommend OWHTO over other procedures for the treatment of degenerative gonarthrosis.

During the treatment period after HTO, patients were evaluated using the JOA score. This scoring method consists of four items: pain on walking, pain going up and down stairs, range of motion, and hydrarthrosis. This study excluded the range of motion and hydrarthrosis scores from the evaluation so that the degree of 
Table 3 Comparison of patients' characteristics between the Early Cured group and the Late Cured group

\begin{tabular}{lll}
\hline & $\begin{array}{l}\text { Early Cured group } \\
\text { (mean } \pm \text { SE}^{\circ} \text { (range)) }\end{array}$ & $\begin{array}{l}\text { Late Cured group } \\
\text { (mean } \pm \text { SE}^{\circ} \text { (range)) }\end{array}$ \\
\hline Cases & 38 & 9 \\
Age & $59.4 \pm 1.5(35-73)$ & $61.4 \pm 3.4(42-70)$ \\
Sex & 12 males & 1 male \\
& 29 females & 8 females \\
FTA (before surgery) & $178.5 \pm 0.4(173-185)$ & $177.3 \pm 0.9(172-181)$ \\
FTA (after surgery) & $169.0 \pm 0.4(165-174)$ & $168.6 \pm 0.7(166-173)$ \\
\%MA (before surgery) & $24.1 \pm 1.9(0-48)$ & $26.7 \pm 5.2(0-40)$ \\
\%MA (after surgery) & $66.8 \pm 1.6(47-88)$ & $66.2 \pm 2.8(52-77)$ \\
PTS (before surgery) & $10.4 \pm 0.5(5-16)$ & $10.1 \pm 1.3(4-15)$ \\
PTS (after surgery) & $13.8 \pm 0.5(8-21)$ & $13.7 \pm 1.5(8-19)$ \\
BMI & $25.7 \pm 0.5(20.6-32.0)$ & $28.3 \pm 1.6(23.0-37.0)$ \\
Ability score (before & $37.8 \pm 1.7(10-55)$ & $32.2 \pm 2.2(25-40)$ \\
$\quad$ surgery) & & \\
Opening angle & $10.3 \pm 0.4(5.4-14.7)$ & $9.1 \pm 0.6(6.4-11.7)$ \\
Treatment period & $6.3 \pm 3.9$ SD (1.5-12) & \\
$\quad$ (months) & & \\
\hline
\end{tabular}

FTA femorotibial angle, MA mechanical axis, PTS posterior tibial slope, BMI body mass index, SD standard deviation, SE standard error

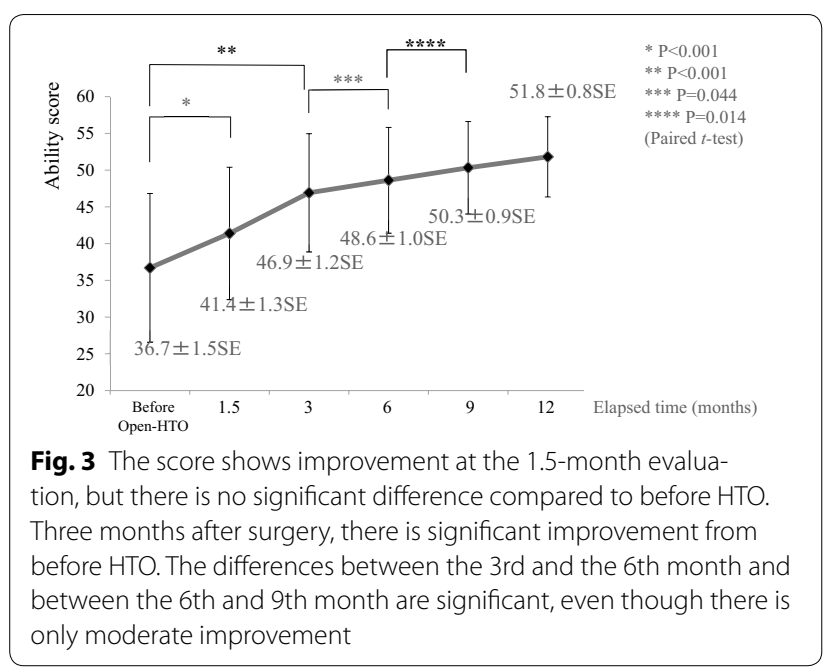

recovery could be evaluated by the patient's subjective symptoms. This score was evaluated over time, and the healing period was considered to have ended when the score stopped showing improvement after OWHTO. Since the condition of the knee joint after HTO is not shown on radiographic images, no imaging evaluation to observe the effect of treatment is available for this technique. Bone fusion of the osteotomy area was assessed by sequential CT images, and it was observed in all cases at the time of implant removal 1 year after surgery.

The results of the present study suggested an average 6-month healing period. In the follow-up examination
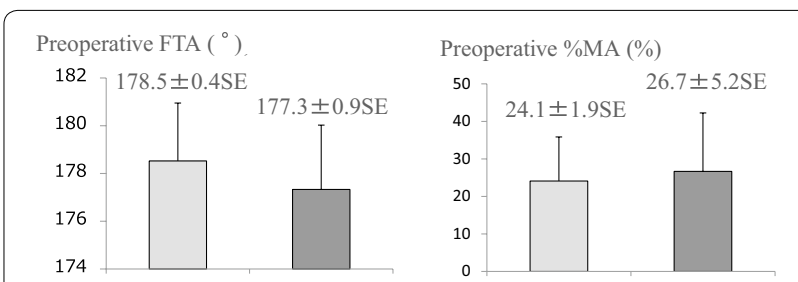

Preoperative PTS $\left({ }^{\circ}\right)$

Preoperative Ability score
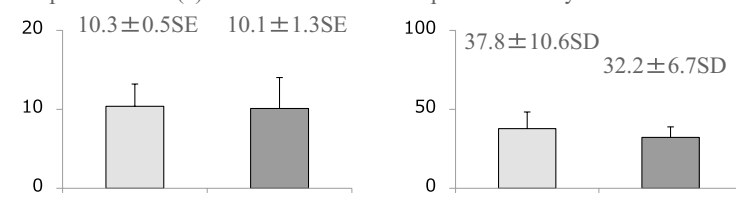

Fig. 4 Comparing the patients' preoperative characteristics, the BMI value is markedly higher in the Late Cured group than in the Early Cured group (Student's $t$ test $P=0.031$ ). There are no other significant differences between the two groups
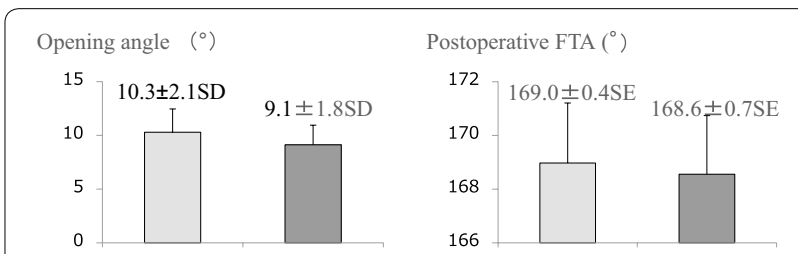

Postoperative PTS $\left({ }^{\circ}\right)$

Postoperative \%MA (\%)
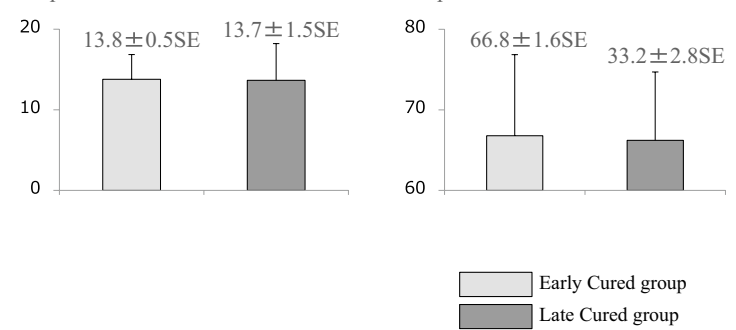

Fig. 5 After the surgery, there are no significant differences in the correction angle, FTA, MA \%, and PTS between the Early Cured group and the Late Cured group

after OWHTO, it is important to describe changes in the patient's condition and the time required for relief of gonalgia. Although good postoperative short-term results are frequently reported, these results are based on improvement or recovery at the end of the examination (Jung et al. 2013; Yim et al. 2013). Takizawa et al. (1995) reported that their patients showed their first improvement 3 months after closed HTO. This improvement in the Ability score was obvious. At the 6-month evaluation, the score was much improved with the increase in muscular strength. In the present study, patients' conditions seemed to be improved 1.5 months after OWHTO (Student's $t$ test, $\mathrm{P}<0.001$ ), but this decreased pain may have 
a Time to healing

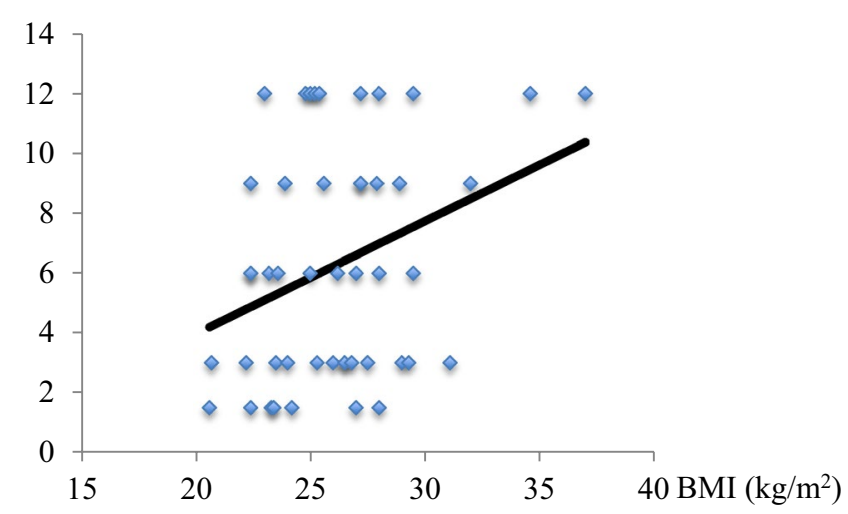

b Time to healing

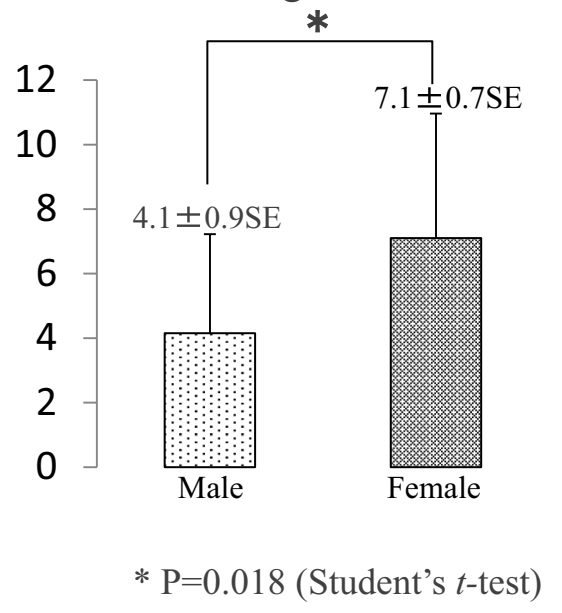

Fig. 6 Factors affecting time to healing (using Pearson's correlation coefficient). a Time to healing and BMI have a positive correlation (correlation coefficient 0.32). b Women take longer to heal than men (Student's $t$ test $P=0.018$ )

come from the low activity due to hospitalization. The difference became significant compared to the preoperative condition 3 months after surgery (Student's $t$ test, $\mathrm{P}<0.001$ ). The examination was carried out on all cases who were discharged from our hospital 3 months after HTO. Judging from these results, 3 months are required before improvement clearly starts, and the average healing period was 6.3 months, which is almost the same as that reported by Takizawa et al.

It is known that ROM, BMI, and other factors affect the long-term results after HTO. PTS, whose value usually increases after OWHTO (Noyes et al. 2005), is one of the major negative factors (Rodner et al. 2006). It has been reported that a high BMI can affect the treatment results. In the present study, a positive correlation was evident between BMI and the healing period. It follows that a high BMI value could also affect the healing period (Spahn et al. 2006). In addition, it has been found that less favorable results are achieved in women, though the reasons for this remain unclear (Niinimäki et al. 2012), and the present results showed the same tendency.

\section{Limitations}

Patients were evaluated until the end of their healing period, up to a maximum of 12 months. This may represent a limitation of this study, as some patients included in the analysis from the Late Cured group were still experiencing pain 12 months after surgery.

It has been reported that some patients have pain caused by the TomoFix plate, but removal of the plate was not performed on any patient in the present study during the 12-month follow-up period. The plate in every case was removed after the 12-month period; it resulted in no apparent symptom improvement, and we think that the TomoFix plate had no effect on the present clinical results.

\section{Conclusion}

In conclusion, this study suggested that the effect of OWHTO appeared in 3 months, and the average healing period was 6.3 months. Patients with a high BMI and women tended to have a longer healing period. These facts should be taken into account when choosing the appropriate surgical method and should be shared with patients with medial type degenerative gonarthrosis, because this can be important information for patients to decide whether to undergo OWHTO. Further studies on the process of treatment and related factors are needed to shorten the healing period.

\section{Authors' contributions}

MY conceived the idea, undertook the data collection, conducted the analysis and wrote the article. $\mathrm{YN}$ and $\mathrm{MD}$ were primarily involved with editing the final manuscript. All authors read and approved the final manuscript.

\section{Acknowledgements}

All authors agreed to submit this article.

\section{Competing interests}

The authors declare that they have no competing interests.

Received: 20 April 2015 Accepted: 28 January 2016

Published online: 12 February 2016

\section{References}

Jung WH, Chun CW, Lee JH, Ha JH, Kim JH, Jeong JH (2013) Comparative study of medial opening-wedge high tibial osteotomy using 2 different 
implants. Arthroscopy 29:1063-1071. doi:10.1016/j.arthro.2013.02.020 (Epub 2013 April 26)

Niinimäki TT, Eskelinen A, Mann BS (2012) Survivorship of high tibial osteotomy in the treatment of osteoarthritis of the knee. Finnish Registry-based study of 3195 knees. J Bone Joint Surg Br 94:1517-1521

Noyes FR, Goebel SX, West J (2005) Opening wedge tibial osteotomy. the 3-triangle method to correct axial alignment and tibial slope. Am J Sports Med 33:378-387

Rodner CM, Adams DJ, Diaz-Doran V, Tate JP, Santangelo SA, Mazzocca AD, Arciero RA (2006) Medial opening wedge tibial osteotomy and the sagittal plane: the effect of increasing tibial slope on tibiofemoral contact pressure. Am J Sports Med 34:1431-1441
Spahn G, Kirschbaum S, Kahl E (2006) Factors that influence high tibial osteotomy results in patients with medial gonarthritis: a score to predict the results. Osteoarthr Cartil 14:190-195 (Epub 2005 Nov 4)

Takizawa T, Akizuki S, Yasukawa Y (1995) Effects on improvement in clinical evaluation, muscle strength and centre of gravity after change of alignment by high tibial osteotomy. Knee 2:19-26

Yim JH, Song EK, Seo HY, Kim MS, Seon JK (2013) Comparison of high tibial osteotomy and unicompartmental knee arthroplasty at a minimum follow-up of 3 years. J Arthroplasty 28:243-247. doi:10.1016/j. arth.2012.06.011 (Epub 2012 July 31)

\section{Submit your manuscript to a SpringerOpen ${ }^{\odot}$ journal and benefit from:}

- Convenient online submission

- Rigorous peer review

- Immediate publication on acceptance

- Open access: articles freely available online

- High visibility within the field

- Retaining the copyright to your article

Submit your next manuscript at $\boldsymbol{~ s p r i n g e r o p e n . c o m ~}$ 